\title{
Visfatin is involved in promotion of colorectal carcinoma malignancy through an inducing EMT mechanism
}

\author{
Jing Yang ${ }^{1, *}$, Kun Zhang ${ }^{1, *}$, Haixing Song ${ }^{1, *}$, Mingbo $\mathbf{W u}^{1, *}$, Jingyi $\mathbf{L i}^{1}$, Ziyi Yong ${ }^{2}$, \\ Sheng Jiang ${ }^{3}$, Xi Kuang ${ }^{4}$, Tao Zhang ${ }^{1}$ \\ ${ }^{1}$ School of Biomedical Sciences, Chengdu Medical College, Chengdu, China \\ ${ }^{2}$ School of Pharmacy, Chengdu Medical College, Chengdu, China \\ ${ }^{3}$ School of Basic Medical Sciences, Chengdu Medical College, Chengdu, China \\ ${ }^{4}$ Department of Pharmacology, Key Laboratory of Drug Targeting and Drug Delivery Systems, West China School of Pharmacy, \\ Sichuan University, Sichuan, China \\ "These authors have contributed equally to this work \\ Correspondence to: Xi Kuang, email: kuangxi56@163.com \\ Tao Zhang, email: ztbillcmc@163.com
}

Keywords: visfatin, colorectal cancer, EMT, Snail, Akt/GSK-3ß

Received: December 10, $2015 \quad$ Accepted: March 28, 2016

Published: April 6, 2016

\section{ABSTRACT}

Increasing evidences suggested visfatin, a newly discovered obesity-induced adipocytokine, is involved in promotion of cancer malignancy and correlated with worse clinical prognosis. While its effects and mechanisms on progression of colorectal cancer (CRC) remain unclear. Our clinical data show that visfatin protein is over expressed, positive associated with lymph node metastasis, high-grade tumor, and poor prognosis in 87 CRC patients. The levels of plasma visfatin are significantly upregulated in Stage IV colon cancer. Visfatin can significantly promote the in vitro migration and invasion of CRC cells via induction epithelial mesenchymal transition (EMT). It can increase the expression and nuclear translocation of Snail, a key transcription factor in regulating EMT. While silencing of Snail attenuates visfatin induced EMT. Further studies reveal visfatin can inhibit the association of Snail with GSK-3 $\beta$ and subsequently suppress ubiquitylation of Snail. In addition, visfatin can increase the expression and nuclear translocation of $\beta$-catenin, elevate its binding with Snail promoter, and then increase the transcription of Snail. While inhibitor of PI3K/Akt, LY294002, abolishes visfatin induced up regulation of Snail, Vimentin (Vim), $\beta$-catenin, and phosphorylated GSK-3 $\beta$. In summary, our data suggest that increased expression of visfatin are associated with a more aggressive phenotype of CRC patients. It can trigger the EMT of CRC cells via Akt/GSK-3 $\beta / \beta$-catenin signals.

\section{INTRODUCTION}

The investigation of underlying pathological mechanisms of cancer invasion and metastasis is important to improve the cure rate and life quality of colorectal cancer (CRC) patients. The adipokine visfatin, which is also indentified as the pre-B-cell colony-enhancing factor (PBEF) and nicotinamide phosphoribosyltransferase (NAMPT), is predominantly secreted by visceral fat tissue, particularly by the macrophages $[1,2]$. Studies indicated that visfatin can also enter into the cytosol and nucleus and then promote the progression of many biological behaviors such as inflammation, angiogenesis, energy metabolism, and cell longevity [3-6]. Recently, visfatin has been reported to be associated with tumorigenesis and/or metastasis of many human cancers such as colon, stomach, brain, pancreas, liver, prostate, and breast cancers [7]. Higher visfatin levels in breast cancer are correlated with overall survival and disease-free survival [8]. Literatures also reported that visfatin overexpression can predict poor response of the patients to doxorubicin [9] or fluorouracil [10] based chemotherapy. Visfatin is closely associated with the pathogenesis of colon cancer, therefore it is considered as a novel and promising biomarker of CRC [11]. The visfatin levels in patients with advanced and early CRC cancer were higher than 
in controls (least significant difference test, $\mathrm{P}=0.004$ and 0.013 , respectively) [12]. Therefore, understanding the effects and mechanisms of visfatin on progression of CRC will be important for the development of prevention and treatment strategies.

Epithelial-mesenchymal transition (EMT), which refers to the epithelial cells get the characteristics of mesenchymal cells under specific physiological conditions, is considered as the first and key step for the migration and invasion of cancers including CRC [13]. Cells undergone of EMT will loss cell-cell adhesion and gain migratory and invasive traits. The loss or decrease of E-Cadherin (E-Cad) is considered to be the primary and most important marker of EMT [14]. Transcription factors such as Snail, Slug, Twist and Zeb, which can suppress the expression of E-Cad directly or indirectly, can regulate the progression of EMT [15]. The process of EMT is positively correlated with the progression, metastasis, and drug resistance of CRC [16]. Increasing evidences suggested that visfatin can promote the migration and invasion of breast [17] and ovarian [18] cancer cells. One recent study revealed that visfatin can enhance the migration and invasion of osteosarcoma cells [19]. However, the effects and mechanisms of visfatin on progression and EMT of CRC cells as a cytokine remain unclear.

Our present study revealed that the expression of visfatin is significantly negatively correlated with the prognosis of CRC patients. Further, in vitro studies suggested that visfatin can promote the EMT phenotype of CRC cells via up regulation of Snail through Akt/GSK$3 \beta / \beta$-catenin signals. Our findings indicated that visfatin might be a target to develop anti-cancer drugs for CRC patients.

\section{RESULTS}

\section{The expression profile of visfatin and its correlation with clinicopathologic characteristics of CRC patients}

To date there are very limited data about visfatin and clinical prognosis of CRC patients. Therefore we assessed the clinical roles of visfatin in $\mathrm{CRC}$ by analyzing the protein levels of visfatin in one commercial tissue microarray from $87 \mathrm{CRC}$ patients. We found that the expression of visfatin was stronger in most primary CRC tissue samples than in their normal counterparts (Figure $1 \mathrm{~A} \& 1 \mathrm{~B})$, and elevated visfatin expression can be detected in CRC patients at advanced clinical stage [assessed using the tumor node metastasis (TNM) system] (Figure 1C \& 1D). Kaplan-Meier analysis of all 87 patients demonstrated a statistically significant negative correlation between overall survival (OS) and visfatin expression level $(p<0.001)$ (Figure 1E). Further, statistical analysis revealed that patients whose tumors express increased node metastasis had greater visfatin expression compared with those with low levels of node metastasis $(\mathrm{p}<0.05$, Table 1$)$. We next analyzed the plasma visfatin levels in cancer patients and normal controls. Our results revealed that the concentration of visfatin in health people was significantly $(\mathrm{p}<0.01)$ less than that in CRC patients (Figure 1F). Spearman correlation analysis showed that the plasma visfatin levels were highly correlated with colon cancer stages $(r=0.505, p<0.05)$. After stratification of the cancer patients according to their clinical stages, plasma visfatin was significantly upregulated in Stage IV colon cancer when compared with that in normal controls, Stage I-II, Stage III, and Stage I-III combined. Collectively, our data suggested that increased expression of visfatin resulted in a more aggressive phenotype in CRC patients.

\section{Visfatin triggers the in vitro motility and EMT of CRC cells}

Clinical data revealed that visfatin is positively correlated with lymph node metastasis of CRC patients, we then investigated the effects of visfatin on the in vitro motility of CRC cells. Overexpression of visfatin significantly promoted wound closure of HCT-116 cells (Figure 2A). While the silencing of visfatin by its specific siRNA inhibited the wound closure as compared to the control group (Figure 2B). Boyden chambers were used to confirm the roles of visfatin on the in vitro invasion of CRC cells. As shown in Figure 2C, the number of invaded HCT-116 and SW480 cells transfected with visfatin was significantly $(\mathrm{p}<0.01)$ greater than that of the control cells.

The increased motility of CRC cells suggested visfatin may modulate the EMT process, of which the epithelial makers E-cad is down-regulated, whereas the mesenchymal markers $\mathrm{N}$-cad and Vim are up-regulated [20]. The EMT of CRC cells was observed after stimulation with visfatin for $48 \mathrm{~h}$. Cells resulted in a significant change in morphology, from cobblestone morphology to mesenchymal spindle-like and fusiform features (Figure S1). Over expression of visfatin obviously down regulated the expression of E-Cad, while increased the expression of Vim and N-Cad in both HCT-116 and SW480 cells (Figure 2D). Similarly, results of mRNA expression profiles of EMT markers in HCT-116 cells also confirmed that over expression of visfatin triggered EMT (Figure 2E). Further, the silencing of visfatin by siRNA exhibited opposite effects in HCT-116 cells (Figure 2F \& 2G). Collectively, these observations showed a critical role of visfatin in the EMT and metastatic phenotypes of CRC cells.

\section{Visfatin triggers the EMT of CRC cells via up regulation of Snail}

Since transcription factors Snail plays a critical role in regulating EMT [21], we then investigated whether visfatin can promote the expression and nuclear 
translocation of Snail. Results showed that both protein and mRNA expression of Snail were significantly up regulated in CRC cells transfected with visfatin construct (Figure $3 \mathrm{~A})$. While, the protein and mRNA expression of Snail were obviously down regulated in HCT-116 and SW480 cells transfected with si-visfatin (Figure 3B). Western blot analysis showed that visfatin can increase the expression of Snail in CRC cells via a dose dependent manner (Figure
$3 C)$. Further, the sub cellular localization of Snail in visfatin $(200 \mathrm{ng} / \mathrm{ml})$ treated HCT-116 cells were checked by use of immunofluorescence staining. The result showed that visfatin treatment obviously triggered the nuclear translocation of Snail in HCT-116 cells (Figure 3D).

To verify the roles of Snail in visfatin induced EMT of CRC cells, HCT-116 and SW480 cells were transfected with non-targeting control si-RNA or si-Snail for $24 \mathrm{~h}$,
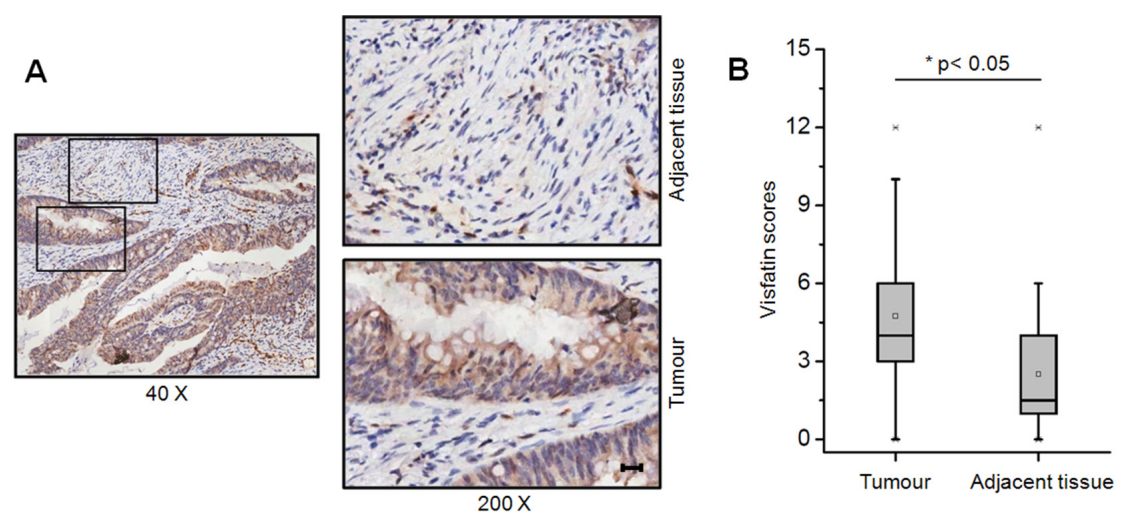
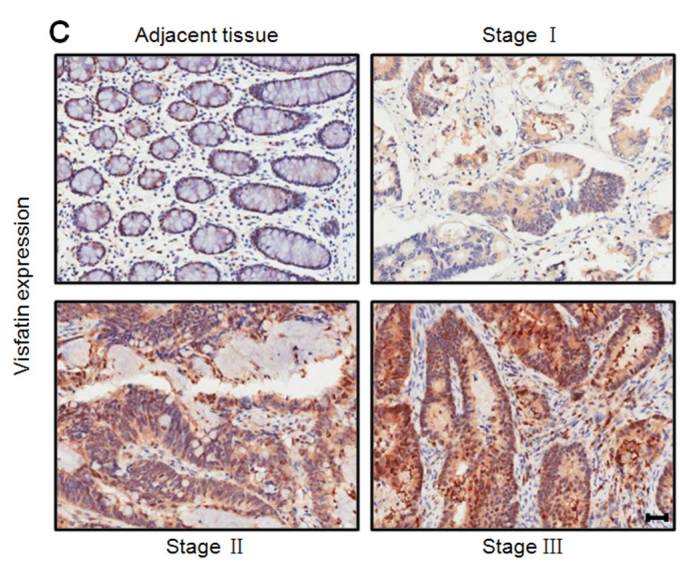

E

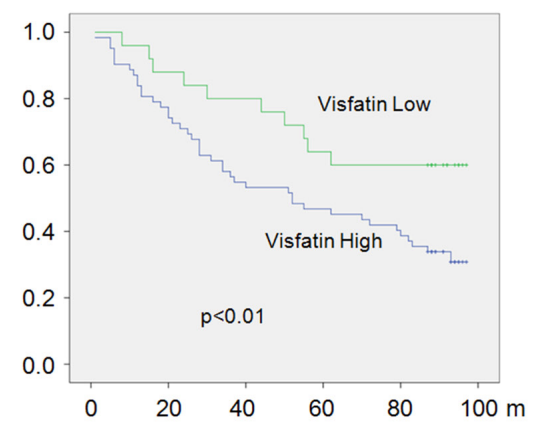

D

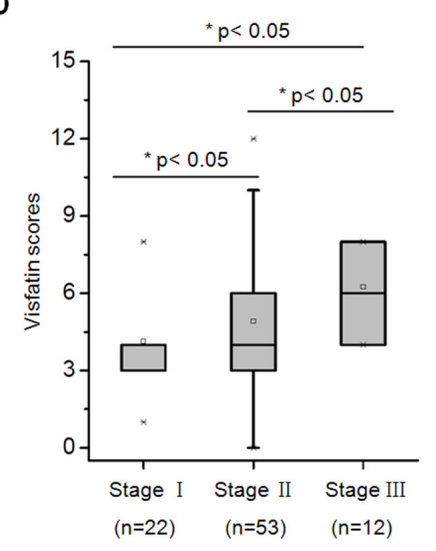

F

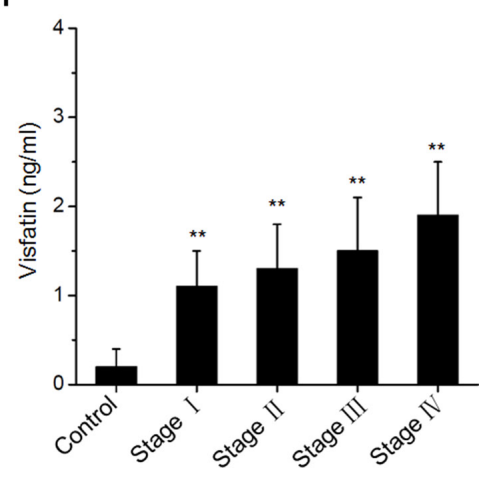

Figure 1: Visfatin expression in clinical samples of colorectal cancer patients. A. Typical immunohistochemical staining for visfatin expression in a tumor and its adjacent tissue from a CRC patient, scale bar is $20 \mu \mathrm{m}$; $\mathbf{B}$. Scores for visfatin staining in paired, adjacent normal and tumor tissue samples obtained from different CRC patients by immunohistochemical staining; C. Typical staining for visfatin in tumor samples from CRC patients with different TNM stages, using immunohistochemical staining, scale bar is $100 \mu \mathrm{m}$; $\mathbf{D}$. Scores for visfatin staining in tumor samples from CRC patients with different TNM stages by immunohistochemical staining; E. Visfatin expression is negatively correlated with prognosis in CTC patients. Overall survival (OS) in patients with high/medium levels of visfatin $(\mathrm{n}=62)$ vs the remaining patients $(\mathrm{n}=25)$ was plotted by the Kaplan-Meier method; F. The content of visfatin $(\mathrm{ng} / \mathrm{ml})$ in health $(\mathrm{n}=200)$ and different TNM stages CRC patients ( $\mathrm{n}=50$ for each stage). 
Table 1: Visfantin expression in $87 \mathrm{CRC}$ patients

\begin{tabular}{|c|c|c|c|c|}
\hline Characteristics & $\mathbf{N}$ & Visfatin Low/No & $\begin{array}{c}\text { Visfatin High/ } \\
\text { Medium }\end{array}$ & $p$ value \\
\hline \multicolumn{5}{|l|}{ Age } \\
\hline$\leq 60$ & 16 & 6 & 10 & 0.391 \\
\hline$>60$ & 71 & 19 & 52 & \\
\hline \multicolumn{5}{|l|}{ Gender } \\
\hline Male & 46 & 12 & 34 & 0.563 \\
\hline Female & 41 & 13 & 28 & \\
\hline \multicolumn{5}{|l|}{ Tumor size } \\
\hline$\leq 10 \mathrm{~cm}^{3}$ & 29 & 8 & 21 & 0.866 \\
\hline$>10 \mathrm{~cm}^{3}$ & 58 & 17 & 41 & \\
\hline \multicolumn{5}{|l|}{$\begin{array}{l}\text { Node metastasis } \\
(n=78)\end{array}$} \\
\hline Negative $(<10)$ & 24 & 12 & 12 & 0.023 \\
\hline Positive $(\geq 10)$ & 54 & 13 & 41 & \\
\hline
\end{tabular}

and then treated with visfatin $(200 \mathrm{ng} / \mathrm{ml})$ for $48 \mathrm{~h}$. The silencing of Snail was confirmed by qRT-PCR and Western blot analysis (Figure 3E). The results revealed that silencing of Snail by both si-Snail2 (Figure 3F) and si-Snail1 (Figure S2) significantly attenuated visfatin induced down regulation of E-cad and up regulation of Vim and N-Cad in CRC cells. It suggested that Snail is essential for visfatin induced EMT in CRC cells.

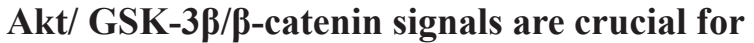 visfatin induced Snail up regulation}

To investigate the molecular mechanisms underlying visfatin induced Snail up regulation, inhibitors of NFкB (BAY11-7082), PI3K/AKT (LY294002), ERK1/2 (PD98059), and p38 (SB-203580) were used, since visfatin has been reported to activate these signals [22]. The results showed that PI3K/AKT inhibitor (LY294002), while not the others, obviously blocked visfatin induced Snail expression (Figure 4A). It suggested that activation of the PI3K/AKT pathway is responsible for visfatin mediated Snail over expression.

GSK-3 $\beta / \beta$-catenin signals which act as the downstream of PI3K/AKT pathway can regulate Snail during EMT [23-25]. GSK-3 $\beta$ maintains an active state in dephosphorylated form, while $\beta$-catenin is complexed with and phosphorylated by GSK-3 $\beta$ for the ubiquitination and the following proteasome mediated degradation [25]. To investigate whether GSK-3 $\beta / \beta$-catenin signals were involved in visfatin induced Snail up regulation, we treated HCT-116 cells with LY294002 prior to visfatin treatment, and then the expression of p-AKT(Ser473), p-GSK-3 $\beta$ (Ser9), $\beta$-catenin, and Snail were determined by Western blot analysis. The results showed that the levels of p-AKT, p-GSK-3 $\beta$, $\beta$-catenin, and Snail were increased after visfatin treatment at $6 \mathrm{~h}$, while these effects were reversed upon treating with LY294002 alone or in combination with visfatin (Figure 4B). Further, we found that visfatin treatment can increase the levels of $\mathrm{p}-\mathrm{GSK}$ $3 \beta$ and $\beta$-catenin in both HCT-116 and SW480 cells via a time dependent manner (Figure $4 \mathrm{C}$ ). To further verify the roles of GSK-3 $\beta$, we pretreated HCT-116 cells with or without $\mathrm{LiCl}$ (a potent GSK-3b inhibitor) for $1 \mathrm{~h}$ and then further treated with visfatin for $6 \mathrm{~h}$, the results showed that inhibition of GSK-3 $\beta$ markedly elevated the levels of Snail expression, while visfatin induced Snail up regulation was not further elevated in the presence of $\mathrm{LiCl}$ (Figure 4D). Further, we knocked down the expression of $\beta$-catenin in HCT 116 cells. The results showed that both si- $\beta$-catenin 1 (Figure 4E) and si- $\beta$-catenin2 (Figure S3) significantly attenuated visfatin induced Snail up regulation. This was confirmed by the results of immunofluorescence staining that visfatin treatment can increase the expression and nuclear localization of $\beta$-catenin in HCT-116 Cells (Figure S4). Taken together, these results demonstrated that visfatin up-regulated Snail in CRC cells by activating Akt/GSK-3 $\beta / \beta$-catenin signaling.

\section{The roles of GSK-3 $\beta / \beta$-catenin in visfatin induced Snail up regulation}

Recent studies indicated GSK-3 $\beta / \beta$-catenin can up regulate Snail expression via transcriptional and posttranscriptional modification [26, 27]. Considering that ubiquitin mediated proteasomal degradation processes are critical for protein stability of Snail, we measured 
the ubiquitination state of Snail in HCT-116 cells treated with visfatin or proteasome inhibitor MG132 for $12 \mathrm{~h}$. The results showed that visfatin obviously suppressed the ubiquitylation of Snail as compared with MG132, although total stabilized Snail proteins were parallel (Figure 5A). Previous studies indicated that GSK-3 $\beta$ can phosphorylate Snail and then induce the protein degradation of Snail [23], then we examined the association of Snail and GSK$3 \beta$. The results showed that the association of Snail with GSK-3 $\beta$ was decreased in cells treated with visfatin, compared with cells treated with MG-132 (Figure 5B). Similarly, when GSK-3 $\beta$ was immunoprecipitated from HCT116 cells, the associated Snail was markedly
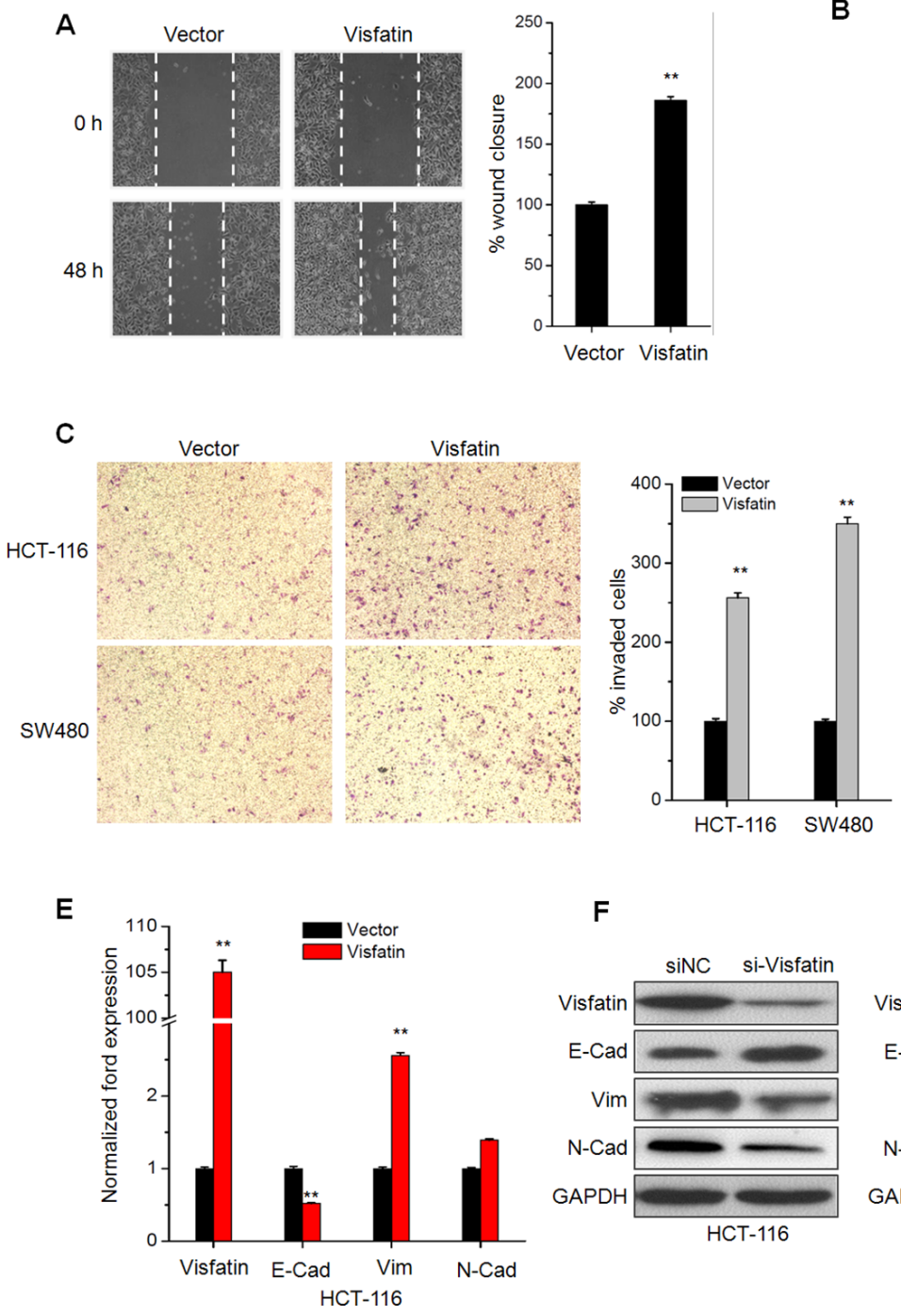

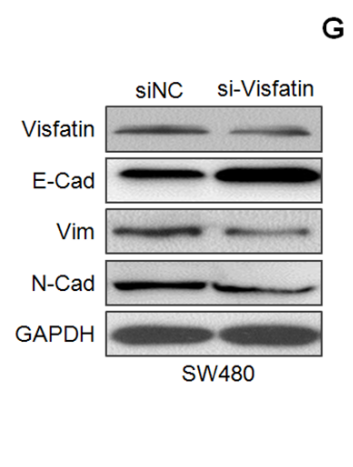

decreased in cells treated with visfatin as compared with MG-132. These results suggested that visfatin can inhibit the association of Snail with GSK-3 $\beta$ and subsequently suppressed ubiquitylation of Snail.

It was suggested that $\beta$-catenin can directly activate the transcription of Snail [27]. Then, we determined if visfatin treatment influenced the binding of $\beta$-catenin subunits on the SNAIL promoter. ChIP assays showed that significant $\beta$-catenin occupancy at Snail promoter when treated with visfatin (Figure 5C). Further, the silencing of $\beta$-catenin decreased the transcription of Snail and attenuated visfatin induced up regulation of Snail transcription in HCT-116 cells (Figure 5D). Collectively,

B
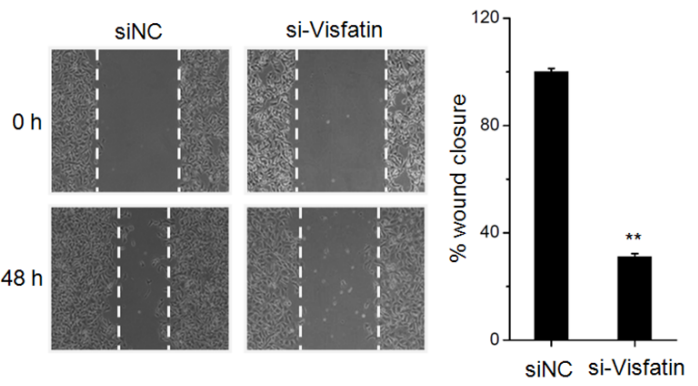

D

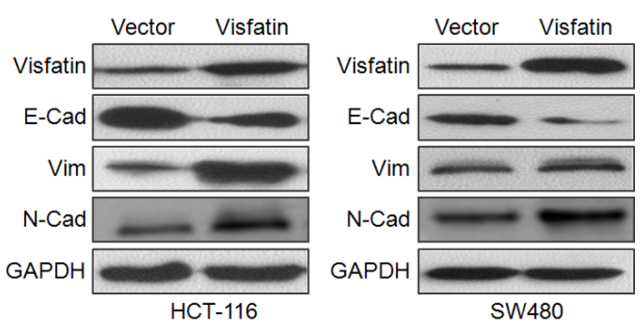

G

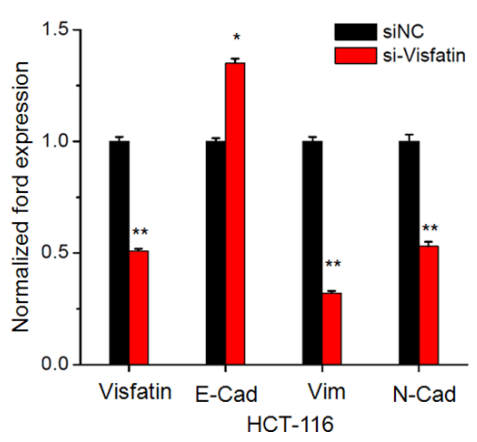

Figure 2: Visfatin triggers the in vitro motility and EMT of CRC cells. A. Confluent monolayers of HCT-116 cells transfected with vector control or visfatin construct for $24 \mathrm{~h}$ were scraped by a pipette tip to generate wounds and then were cultured for $24 \mathrm{~h}$. Representative images of wounds and the statistic results at each concentration were recorded. B. Would healing assays for HCT-116 cells transfected with siNC or si-visfatin for $24 \mathrm{~h}$; C. HCT-116 or SW480 cells were transfected with vector control or visfatin construct for $24 \mathrm{~h}$ and then allowed to invade spread through the matrix gel and into the under-side of the filter for $24 \mathrm{~h}$. The number of invaded cells were fixed, stained, photographed, and compared with the control group. D and F. HCT-116 or SW480 cells were transfected with vector control, visfatin construct, siNC control or si-visfatin for $48 \mathrm{~h}$ as indicated, then the protein levels of visfatin, E-cad, Vim, and N-Cad were analyzed by Western blot analysis. E and G. HCT-116 cells were transfected with visfatin construct or si-visfatin for $24 \mathrm{~h}$ as indicated the mRNA levels of visfatin, E-cad, Vim, and N-Cad were analyzed by qRT-PCR. Scale bar is $100 \mu \mathrm{m},{ }^{*} \mathrm{p}<0.05$ compared with control, ${ }^{* *} \mathrm{p}<0.01$ compared with control. 
our data revealed that visfatin can increase the expression of $\beta$-catenin, elevate its binding with Snail promoter, and then increase the transcription of Snail.

\section{DISCUSSION}

Increased evidences suggested visfatin can promote the development of $\mathrm{CRC}$ and other cancers such as breast, prostate, and endometrial cancers [28, 29]. Recently, limited literatures indicated that visfatin might promote the EMT of cancer cells as a soluble

A
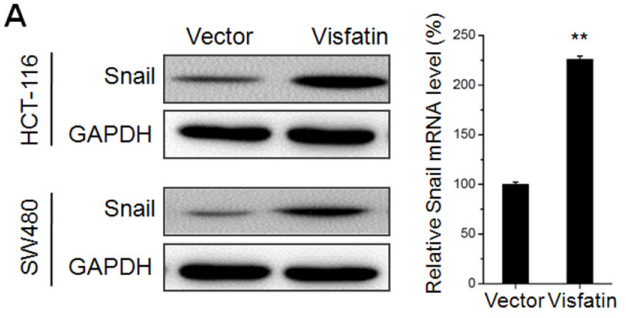

C
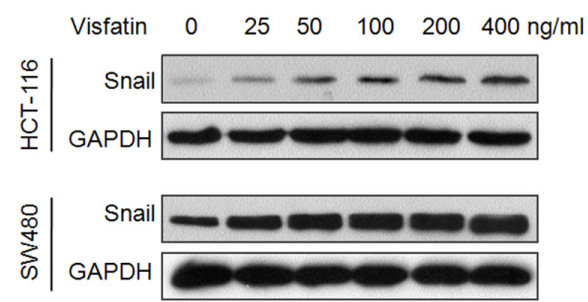

E

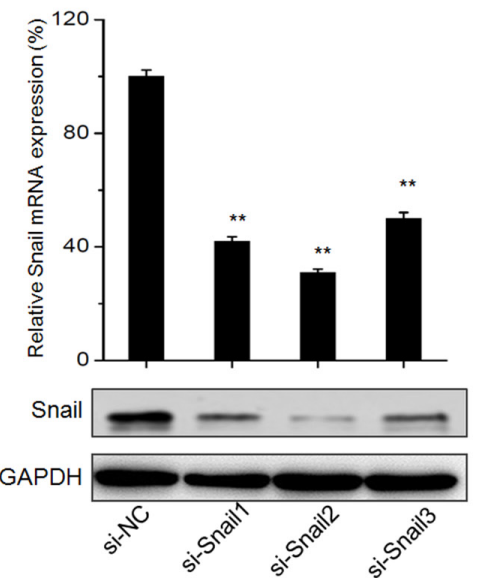

$\mathbf{F}$

(1)

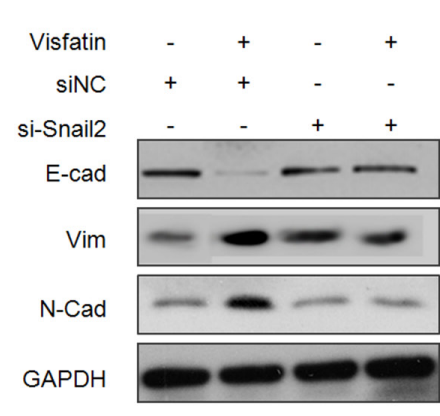

HCT-116

(1) factor independent of its enzymatic activity [19, 30]. Our present study revealed that increased expression of visfatin resulted in a more aggressive phenotype in CRC patients. Further, visfatin can increase the in vitro migration and invasion of CRC cells via induction of EMT. Snail, an important transcription factor of EMT, mediated visfatin induced EMT of CRC cells. Akt/ GSK-3 $\beta / \beta$-catenin signals were crucial for visfatin induced Snail up regulation. Generally, the present study revealed that visfatin can be considered as a therapy target for CRC.

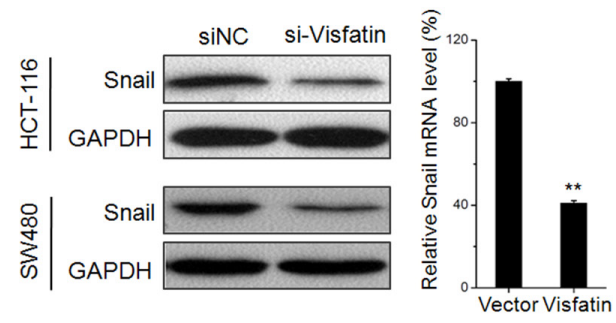

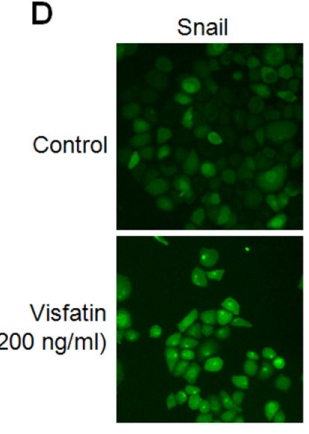
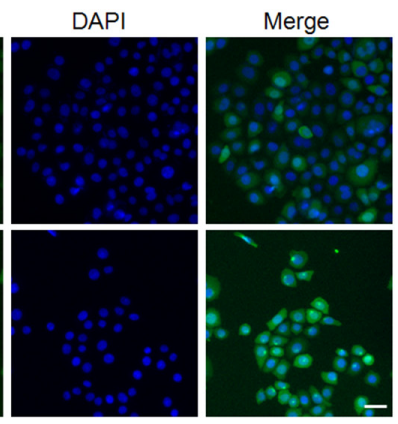

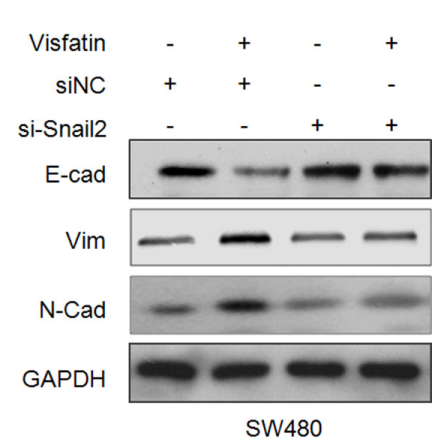

SW480

Figure 3: Visfatin triggers the EMT of CRC cells via up regulation of Snail. A. HCT-116 or SW480 cells were transfected with vector control or visfatin construct for $24 \mathrm{~h}$, then the protein or mRNA levels of Snail were analyzed by Western blot analysis or RT-PCR, respectively; B. HCT-116 or SW480 cells were transfected with siNC or si-visfatin for $24 \mathrm{~h}$, then the protein or mRNA levels of Snail were analyzed by Western blot analysis or RT-PCR, respectively; C. HCT-116 or SW480 cells were treated with increasing concentrations of visfatin for $24 \mathrm{~h}$, the levels of Snail were checked by Western blot analysis; D. HCT-116 cells were treated with $200 \mathrm{ng} / \mathrm{ml}$ visfatin for 24 $\mathrm{h}$, the cellular location of Snail (green) were examined by immunofluorescence staining and nuclei were stained with DAPI (blue), scale bar is $20 \mu \mathrm{m}$; E. HCT-116 cells were transfected with Snail specific si-RNA (si-Snail1 3) or negative control si-RNA (si-NC) for 24 h, and then the mRNA and protein expression of Snail were analyzed by qRT-PCR and Western-blot, respectively; F. HCT-116 or SW480 cells transfected with Snail specific si-RNA (si-Snail2) or negative control si-RNA (si-NC) for $24 \mathrm{~h}$ and then exposed to $200 \mathrm{ng} / \mathrm{ml}$ visfatin for another $48 \mathrm{~h}$, the expression of EMT related markers were measured by use of Western blot analysis. $* * p<0.01$ compared with control. 
To the best of our knowledge, this is the first systematic study to evaluate the prognostic significance of visfatin expression in CRC patients. A significant increase in visfatin expression was observed in colorectal cancer tissues. Furthermore, visfatin is an indicator of poor prognosis in colorectal cancer for both plasma concentrations and immunohistochemical staining of tumor biopsies. As a newly discovered obesity-induced adipocytokine, Dalamaga et al. observed that the mean serum visfatin level was significantly higher in breast cancer than in controls or patients with benign breast lesions [31]. As to $\mathrm{CRC}$, the visfatin levels in patients with advanced and early $\mathrm{CRC}$ cancer were higher than

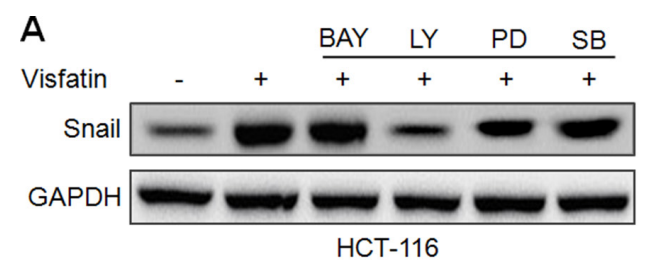

B

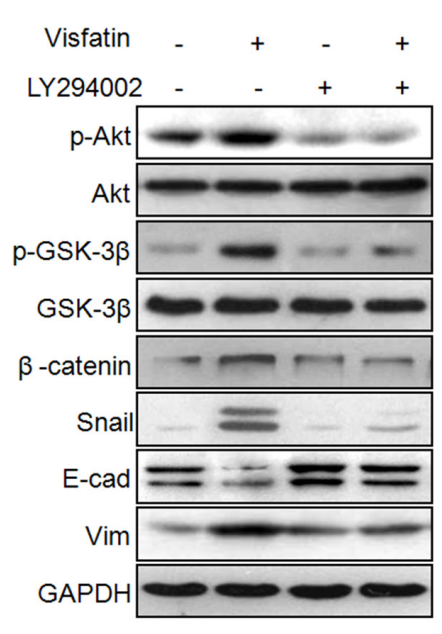

D

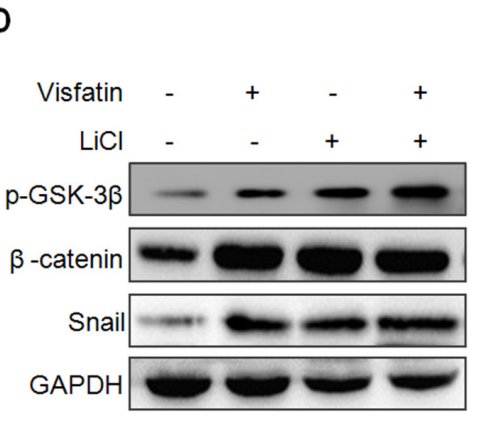

C
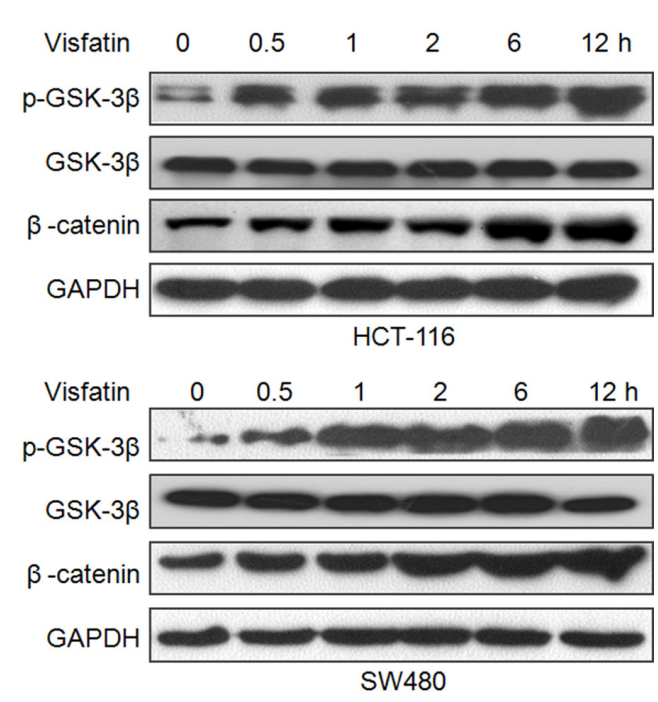

in controls [12]. The plasma visfatin levels can provide potential biomarkers for predicting early and advanced CRC and yield a ROC curve area of 72 and $86 \%$, respectively [12]. This was supported by other studies that visfatin levels were significantly higher in CRC patients than that in controls $(\mathrm{p}<0.01)[11,32]$. Our present study revealed that primary tumor tissue visfatin level was associated with the clinicopathologic stage. Generally, our observation is in agreement with previous reports that higher visfatin expression is observed in primary colorectal cancer than in nonneoplastic mucosa.

Our study revealed that visfatin treatment can trigger the EMT of CRC cells via up regulation of Snail.

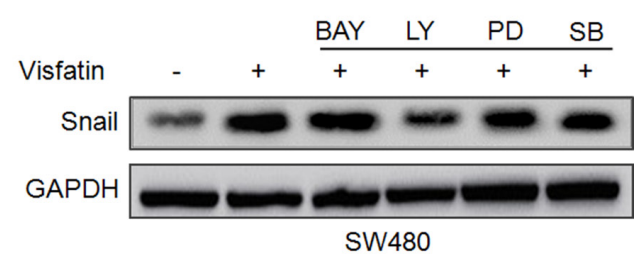

$\mathbf{E}$

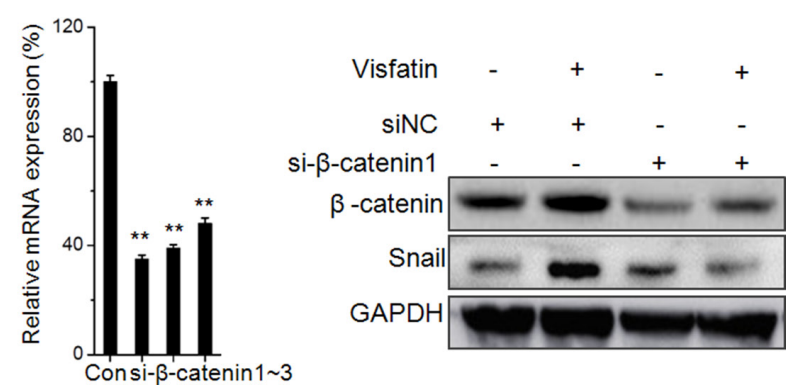

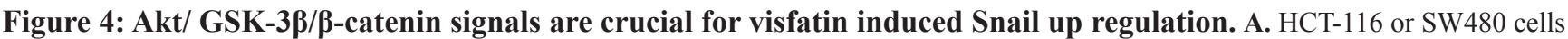
were pretreated with inhibitors of NF-kB (BAY11-7082, BAY), PI3K/AKT (LY294002, LY), ERK1/2 (PD98059, PD), and p38 (SB$203580, \mathrm{SB}$ ) for $1 \mathrm{~h}$, and then treated with visfatin for $12 \mathrm{~h}$; B. HCT-116 cells were pretreated with LY294002 for $1 \mathrm{~h}$, and then treated with visfatin for $12 \mathrm{~h}$; C. HCT-116 or SW480 cells were treated with visfatin for increasing time periods, the expression of p-GSK-3 $\beta$ and $\beta$-catenin were checked by Western blot analysis; D. HCT-116 cells were pretreated with LiCl for $1 \mathrm{~h}$, and then treated with visfatin for 12 h; E. HCT-116 cells were transfected with si- $\beta$-catenin 1 or si-NC for $24 \mathrm{~h}$, and then treated with visfatin for $12 \mathrm{~h} .{ }^{* *} p<0.01$ compared with control. 
Soncini et al showed visfatin over expression is sufficient to induce EMT in mammary epithelial cells via elevation the expression of TGF- $\beta$ [30]. Visfatin also can promote the EMT of osteosarcoma cells via the NF- $\mathrm{B} / \mathrm{Snail}-1 /$ EMT pathway [19]. Several transcription factors have been implicated in the progression of EMT. Our present study revealed that visfatin treatment obviously increased the expression and nuclear translocation of Snail in CRC cells, while Snail knockdown attenuated visfatin induced EMT. It confirmed that Snail is essential for visfatin induced EMT in CRC cells. This is also consistent with recent study that visfatin can increase both protein an mRNA levels of Snail in osteosarcoma cells [19].

Several downstream pathways including PI3K/ Akt and NF- $\kappa \mathrm{B}$ have been reported to contribute to visfatin-induced targeted gene transcription [28, 33]. We found that the inhibitor of PI3K/Akt, while not NF- $\mathrm{BB}$, ERK1/2, or p38-MAPK, attenuated visfatin induced up regulation of Snail and EMT progression. The activation of Akt/GSK-3 $\beta$ signal has been reported to be associated with a loss of cell adhesion, increased of cell motility, and poor prognosis of CRC [34]. GSK-3 $\beta$ can decrease the ubiquitination and proteasomal degradation of Snail $[23,35]$. Further, inactivation of GSK-3 $\beta$ can upregulate $\beta$-catenin during gastrin [36] and TNF- $\alpha$ [37] induced EMT of cancer cells. Our study revealed that visfatin can up regulate the expression of Snail via both transcriptional and post-transcriptional modification though Akt/ GSK-3 $\beta / \beta$-catenin signals. Firstly, visfatin increases Snail expression by increasing Snail protein stability particularly through phosphorylation of GSK-3 $\beta$ and then inhibition their association. Secondly, visfatin can elevate

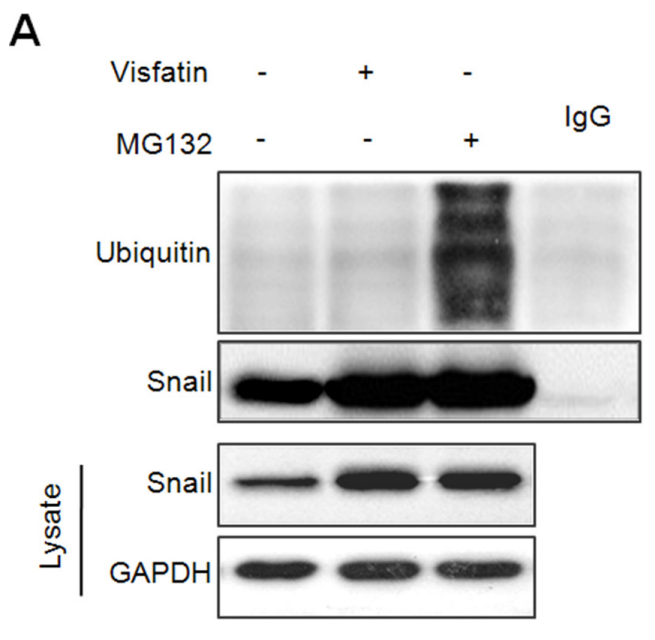

B
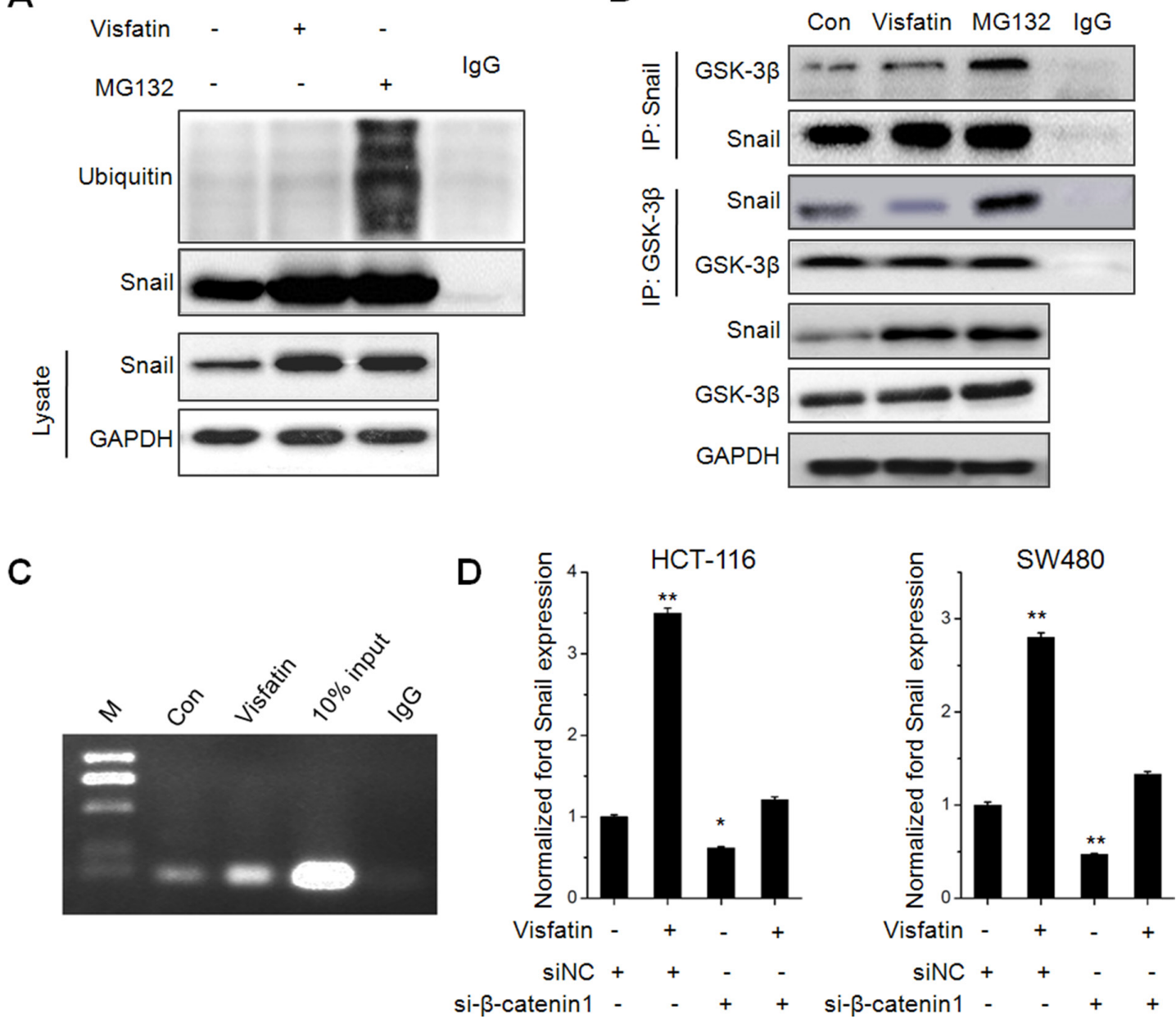

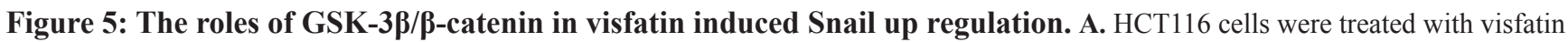
$(200 \mathrm{ng} / \mathrm{ml})$ or MG132 (10 mM) for $12 \mathrm{~h}$, After Snail was immunoprecipitated from equal amount of lysates (two lower panels), the ubiquitination of Snail was examined by Western blot analysis; B. HCT116 cells were treated with visfatin (200 ng/ml) or MG132 (10 $\mathrm{mM}$ ) for $12 \mathrm{~h}$, Snail or GSK-3 $\beta$ were immunoprecipitated respectively from equal amount of lysates and the associated GSK-3 $\beta$ or Snail were detected by Western blot analysis; C. HCT-116 cells were treated with visfatin $(200 \mathrm{ng} / \mathrm{ml})$ or MG132 (10 mM) for $12 \mathrm{~h}$, and then the recruitment of $\beta$-catenin to Snail promoter was determined by ChIP. Immunoprecipitated products were amplified by Real-time PCR; D. HCT-116 or SW480 cells were transfected with siNC or si- $\beta$-catenin 1 for $24 \mathrm{~h}$, and then further treated with visfatin $(200 \mathrm{ng} / \mathrm{ml})$ for $12 \mathrm{~h}$, the mRNA levels of Snail were measured by Real-time PCR. ${ }^{*} p<0.05$ compared with control; ** $p<0.01$ compared with control. 
the binding of $\beta$-catenin subunits on the SNAIL promoter and then promote the transcription of Snail [27]. Although the exact role of GSK-3 $\beta / \beta$-catenin in cancer metastasis remains controversial and is likely cell type- and stimulusdependent, our study revealed that Akt/GSK-3 $\beta / \beta$-catenin is crucial for visfatin induced EMT of CRC cells.

In summary, we have identified that visfatin can trigger the EMT of CRC cells via Akt/GSK-3 $\beta / \beta$-catenin signals and suggested that increased expression of visfatin resulted in a more aggressive phenotype in CRC patients. Though the precise mechanism remains undetermined, these findings will help to better understand the roles of visfatin on the progression of $\mathrm{CRC}$ and indicate that visfatin might be a valuable target for CRC therapy.

\section{MATERIALS AND METHODS}

\section{Patients plasma samples and visfatin measurement}

The study was approved by the Ethical Committee of Chengdu Medical College. Plasma samples were collected to investigate the concentration of plasma visfatin with the clinicopathologic characteristics of CRC patients . 200 plasma samples from CRC patients and 100 plasma samples from healthy individuals were obtained from The First Affiliated Hospital of Chengdu Medical College (Sichuan province, China) between 2005 to 2012. Pathologic classification of disease in all patients was performed following the International Union Against Cancer (UICC) and American Joint Committee on Cancer (AJCC) TNM staging system for colon cancer established in 2003. Blood samples were collected from all patients before operation and therapy. Visfatin levels in plasma were measured by standard enzyme immunoassay.

\section{Immunohistochemistry and scoring}

Commercial tissue microarray (TMAs; HColAde180Sur-06, Shanghai Outdo Biotech, Shanghai, China) from $90 \mathrm{CRC}$ patients with paired normal mucosal counterparts were used to evaluate the expression of visfatin protein. Immunohistochemistry using a visfatin antibody was performed as previously described [38]. Briefly, antigen retrieval was performed using high pressure for $5 \mathrm{~min}$, using citrate buffer, $\mathrm{pH}$ 6.0. The sections were incubated overnight with the primary antibodies at $4{ }^{\circ} \mathrm{C}$. Negative controls were performed by replacing the primary antibody with PBS. Finally, the slides were analyzed separately by two pathologists without knowing the patients' clinical information. The staining intensity was scored on a scale of $0-3$ as negative (0), weak (1), medium (2) or strong (3). The extent of the staining, defined as the percentage of positive staining areas of tumor cells in relation to the whole tumor area, was scored on a scale of $0(0 \%), 1(1-25 \%), 2(26-50 \%), 3$
$(51-75 \%)$ and $4(76-100 \%)$. An overall protein expression score (overall score range, 0-12) was calculated by multiplying the intensity and positivity scores according to previous study [39].

\section{Cell culture and transfection}

Human CRC cell HCT-116 and SW480 cells were purchased from the American Type Culture Collection (Manassas, VA, USA), maintained in our laboratory, and cultured in RPMI 1640 or DMEM medium (Invitrogen Corporation, Carlsbad, CA, USA) supplemented with $10 \%$ heat-inactivated fetal Bovin serum, $100 \mathrm{U} / \mathrm{ml}$ penicillin, and $10 \mu \mathrm{g} / \mathrm{ml}$ streptomycin at $37{ }^{\circ} \mathrm{C}$ in a $5 \%$ $\mathrm{CO}_{2}$ atmosphere. An ABI 3130 Genetic Analyzer (Applied Biosystems) was used for the profiling. The DNA profile data was cross-checked with the ATCC data bank. For transfection, cells were seeded into plates and transfected with pEGFP-N1 (vector control), pEGFR-N1/visfatin (Visfatin construct), siRNA negative control (si-NC), siSnail, si-visfatin, or si- $\beta$-catenin by use of Lipofectamine 2000 reagent (Invitrogen).

\section{In vitro wound-healing and transwell invasion assay}

A wound-healing assay was used to compare the migratory ability of HCT-116 and SW480 cells as described previously [40]. The cell migration and invasion assay was using 6-well transwell plates (Falcon cell culture inserts, 8 - $\mu \mathrm{m}$ pore size, BD, NJ) according to previous study [41].

\section{Western blotting analysis}

Western blotting was performed as previously described [42].

\section{Quantitative real-time PCR}

After treatment as indicated, total mRNA of cells was extracted with TRIZOL reagent. First strand of cDNA was generated from $2 \mu \mathrm{g}$ total RNA using oligo-dT primer and Superscript II Reverse Transcriptase (GIBCO BRL, Grand Island, NY, USA). Quantitative Real-Time PCR was run on an iCycler (Bio-Rad, Hercules, USA) using validated primers and SYBR Premix Ex Taq II (Takara, Japan) for detection. The cycle number when the fluorescence first reached a preset threshold $(\mathrm{Ct})$ was used to quantify the initial concentration of individual templates for expression of mRNA of genes of interest. Transcripts of the housekeeping gene GAPDH in the same incubations were used for internal normalization. Primer pairs were as follows: visfatin, forward 5'- GCC AGC AGG GAA TTT TGT TA-3' and reverse 5'- TGA TGT GCT GCT TCC AGT TC-3'; E-Cad forward 5'- TAC ACT GCC CAG GAG CCA GA -3' and reverse 5'- TGG CAC CAG TGT 
CCG GAT TA -3'; Vim forward 5'- TGA GTA CCG GAG ACA GGT GCA G -3' and reverse 5'- TAG CAG CTT CAA CGG CAA AGT TC-3'; N-Cad forward 5'- GAC GGT TCG CCA TCC AGA C-3' and reverse 5'- TCG ATT GGT TTG ACC ACG G-3'; Snail, forward 5'- GAC CAC TAT GCC GCG CTC TT $-3^{\prime}$ and reverse 5'- TCG CTG TAG TTA GGC TTC CGA TT -3'; $\beta$-Catenin, forward 5'- TCC CTG AGA CGC TAG ATG AGG -3' and reverse 5'- CGT TTA GCA GTT TTG TCA GCT C -3'; GAPDH, forward 5'-GCA CCG TCA AGG CTG AGA AC-3' and reverse 5'-TGG TGA AGA CGC CAG TGG A-3'.

\section{Immunofluorescence}

Immunofluorescence staining was carried out as described previously [43].

\section{Statistical analysis}

All values were reported as mean $\pm \mathrm{SD}$ of three independent experiments unless otherwise specified. Data were analyzed by two-tailed unpaired Student's t-test between two groups and by One-Way ANOVA followed by Bonferroni test for multiple comparison involved. The survival curves were plotted by using Kaplan-Meier analysis. Statistical analysis was carried out using SPSS 16.0 for Windows. A $p$-value of $<0.05$ was considered to be statistically significant.

\section{ACKNOWLEDGMENTS}

This research was supported by the National Natural Science Foundation of China (Grant No. 81301854, 81301919 and 81302205$)$, and all support is gratefully acknowledged.

\section{CONFLICTS OF INTEREST}

The authors declare no conflicts of interest.

\section{REFERENCES}

1. Curat CA, Wegner V, Sengenes C, Miranville A, Tonus C, Busse R, Bouloumie A. Macrophages in human visceral adipose tissue: increased accumulation in obesity and a source of resistin and visfatin. Diabetologia. 2006; 49:744-747.

2. Fukuhara A, Matsuda M, Nishizawa M, Segawa K, Tanaka M, Kishimoto K, Matsuki Y, Murakami M, Ichisaka T, Murakami H, Watanabe E, Takagi T, Akiyoshi M, Ohtsubo T, Kihara S, Yamashita S, et al. Visfatin: A protein secreted by visceral fat that mimics the effects of insulin (Retraction of vol 307, pg 426, 2005). Science. 2007; 318:565-565.

3. Kim SR, Bae SK, Choi KS, Park SY, Jun HO, Lee JY, Jang HO, Yun I, Yoon KH, Kim YJ, Yoo MA, Kim KW,
Bae MK. Visfatin promotes angiogenesis by activation of extracellular signal-regulated kinase 1/2. Biochem Bioph Res Com. 2007; 357:150-156.

4. Bae MK, Su-Ryun K, Soo-Kyung B. Visfatin enhances ICAM-1 and VCAM-1 expression through ROS-dependent NF-kappa B activation in endothelial cells. FEBS J. 2009; 276:187-188.

5. Bae YH, Park HJ, Kim SR, Kim JY, Kang Y, Kim JA, Wee HJ, Kageyama R, Jung JS, Bae MK, Bae SK. Notch1 mediates visfatin-induced FGF-2 up-regulation and endothelial angiogenesis. Cardiovasc Res. 2011; 89:436-445.

6. Ramsey KM, Yoshino J, Brace CS, Abrassart D, Kobayashi Y, Marcheva B, Hong HK, Chong JL, Buhr ED, Lee C, Takahashi JS, Imai SI, Bass J. Circadian Clock Feedback Cycle Through NAMPT-Mediated NAD $(+)$ Biosynthesis. Science. 2009; 324:651-654.

7. $\mathrm{Bi} T Q$, Che XM. Nampt/PBEF/visfatin and cancer. Cancer Biol Ther. 2010; 10:119-125.

8. Li XY, Tang SH, Zhou XC, Ye YH, Xu XQ, Li RZ. Preoperative serum visfatin levels and prognosis of breast cancer among Chinese women. Peptides. 2014; 51:86-90.

9. Folgueira MAAK, Carraro DM, Brentani H, Patra DFD, Barbosa EM, Netto MM, Caldeira JRF, Katayama MLH, Soares FA, Oliveira CT, Reis LFL, Kaiano JHL, Camargo LP, Vencio RZN, Snitcovsky IML, Makdissi FBA, et al. Gene expression profile associated with response to doxorubicin-based therapy in breast cancer. Clin Cancer Res. 2005; 11:7434-7443.

10. Bi TQ, Che XM, Liao XH, Zhang DJ, Long HL, Li HJ, Zhao W. Overexpression of Nampt in gastric cancer and chemopotentiating effects of the Nampt inhibitor FK866 in combination with fluorouracil. Oncol Rep. 2011; 26:1251-1257.

11. Nakajima TE, Yamada $Y$, Hamano $T$, Furuta $K$, Matsuda T, Fujita S, Kato K, Hamaguchi T, Shimada Y. Adipocytokines as new promising markers of colorectal tumors: Adiponectin for colorectal adenoma, and resistin and visfatin for colorectal cancer. Cancer Sci. 2010; 101:1286-1291.

12. Chen M, Wang Y, Li Y, Zhao L, Ye S, Wang S, Yu C, Xie $\mathrm{H}$. Association of plasma visfatin with risk of colorectal cancer: An observational study of Chinese patients. Asia Pac J Clin Oncol. 2013; 12:e65-74.

13. Bates RC, Mercurio AM. The epithelial-mesenchymal transition (EMT) and colorectal cancer progression. Cancer Biol Ther. 2005; 4:365-370.

14. Le Bras GF, Taubenslag KJ, Andl CD. The regulation of cell-cell adhesion during epithelial-mesenchymal transition, motility and tumor progression. Cell Adh Migr. 2012; 6:365-373.

15. Singh A, Settleman J. EMT, cancer stem cells and drug resistance: an emerging axis of evil in the war on cancer. Oncogene. 2010; 29:4741-4751. 
16. Zhu QC, Gao RY, Wu W, Qin HL. Epithelial-mesenchymal Transition and Its Role in the Pathogenesis of Colorectal Cancer. Asian Pac J Cancer Prev. 2013; 14:2689-2698.

17. Park HJ, Kim SR, Kim SS, Wee HJ, Bae MK, Ryu MH, Bae SK. Visfatin promotes cell and tumor growth by upregulating Notch1 in breast cancer. Oncotarget. 2014; 5:5087-5099. doi: 10.18632/oncotarget.2086.

18. Li Y, Li X, Liu KR, Zhang JN, Liu Y, Zhu Y. Visfatin derived from ascites promotes ovarian cancer cell migration through Rho/ROCK signaling-mediated actin polymerization. Eur J Cancer Prev. 2015; 24:231-239.

19. Cheng G, Liu C, Sun X, Zhang L, Liu L, Ouyang J, Li B. Visfatin promotes osteosarcoma cell migration and invasion via induction of epithelial-mesenchymal transition. Oncol Rep. 2015; 34:987-994.

20. Yilmaz M, Christofori G. EMT, the cytoskeleton, and cancer cell invasion. Cancer Metastasis Rev. 2009; 28:15-33.

21. Thiery JP, Acloque H, Huang RYJ, Nieto MA. Epithelialmesenchymal transitions in development and disease. Cell. 2009; 139:871-890.

22. Huang WS, Chen CN, Sze CI, Teng CC. Visfatin induces stromal cell-derived factor-1 expression by betal integrin signaling in colorectal cancer cells. J Cell Physiol. 2013; 228:1017-1024.

23. Zhou BP, Deng J, Xia W, Xu J, Li YM, Gunduz M, Hung MC. Dual regulation of Snail by GSK-3beta-mediated phosphorylation in control of epithelial-mesenchymal transition. Nat Cell Biol. 2004; 6:931-940.

24. Larue L, Bellacosa A. Epithelial-mesenchymal transition in development and cancer: role of phosphatidylinositol 3' kinase/AKT pathways. Oncogene. 2005; 24:7443-7454.

25. Katoh M, Katoh M. Cross-talk of WNT and FGF signaling pathways at GSK3 beta to regulate beta-catenin and SNAIL signaling cascades. Cancer Biol Ther. 2006; 5:1059-1064.

26. Zheng H, Li W, Wang Y, Liu Z, Cai Y, Xie T, Shi M, Wang Z, Jiang B. Glycogen synthase kinase-3 beta regulates Snail and beta-catenin expression during Fas-induced epithelialmesenchymal transition in gastrointestinal cancer. Eur J Cancer. 2013; 49:2734-2746.

27. Gnemmi V, Bouillez A, Gaudelot K, Hemon B, Ringot B, Pottier N, Glowacki F, Villers A, Vindrieux D, Cauffiez C, Van Seuningen I, Bernard D, Leroy X, Aubert S, Perrais M. MUC1 drives epithelial-mesenchymal transition in renal carcinoma through Wnt/beta-catenin pathway and interaction with SNAIL promoter. Cancer Lett. 2014; 346:225-236.

28. Sampath D, Zabka TS, Misner DL, O'Brien T, Dragovich PS. Inhibition of nicotinamide phosphoribosyltransferase (NAMPT) as a therapeutic strategy in cancer. Pharmacol Ther. 2015; 151:16-31.

29. Maldi E, Travelli C, Caldarelli A, Agazzone N, Cintura S, Galli U, Scatolini M, Ostano P, Miglino B, Chiorino G, Boldorini R, Genazzani AA. Nicotinamide phosphoribosyltransferase (NAMPT) is over-expressed in melanoma lesions. Pigment Cell Melanoma Res. 2013; 26:144-146.

30. Soncini D, Caffa I, Zoppoli G, Cea M, Cagnetta A, Passalacqua M, Mastracci L, Boero S, Montecucco F, Sociali G, Lasiglie D, Damonte P, Grozio A, Mannino E, Poggi A, D'Agostino VG, et al. Nicotinamide Phosphoribosyltransferase Promotes Epithelial-toMesenchymal Transition as a Soluble Factor Independent of Its Enzymatic Activity. J Biol Chem. 2014; 289:34189-34204.

31. Dalamaga M, Karmaniolas K, Papadavid E, Pelekanos N, Sotiropoulos G, Lekka A. Elevated serum visfatin/ nicotinamide phosphoribosyl-transferase levels are associated with risk of postmenopausal breast cancer independently from adiponectin, leptin, and anthropometric and metabolic parameters. Menopause. 2011; 18:1198-1204.

32. Nakajima TE, Yamada Y, Hamano T, Furuta K, Gotoda T, Katai H, Kato K, Hamaguchi $\mathrm{T}$ and Shimada $\mathrm{Y}$. Adipocytokine levels in gastric cancer patients: resistin and visfatin as biomarkers of gastric cancer. J Gastroenterol. 2009; 44:685-690.

33. Adya R, Tan BK, Punn A, Chen J, Randeva HS. Visfatin induces human endothelial VEGF and MMP-2/9 production via MAPK and PI3K/Akt signalling pathways: novel insights into visfatin-induced angiogenesis. Cardiovasc Res. 2008; 78:356-365.

34. Pal I, Mandal M. PI3K and Akt as molecular targets for cancer therapy: current clinical outcomes. Acta Pharmacol Sin. 2012; 33:1441-1458.

35. Bachelder RE, Yoon SO, Franci $C$, de Herreros AG, Mercurio AM. Glycogen synthase kinase-3 is an endogenous inhibitor of snail transcription: implications for the epithelial-mesenchymal transition. J Cell Biol. 2005; 168:29-33.

36. Mishra P, Senthivinayagam S, Rana A, Rana B. Glycogen Synthase Kinase-3beta regulates Snail and beta-catenin during gastrin-induced migration of gastric cancer cells. J Mol Signal. 2010; 5:9.

37. Wang H, Wang HS, Zhou BH, Li CL, Zhang F, Wang XF, Zhang G, Bu XZ, Cai SH, Du J. Epithelial-mesenchymal transition (EMT) induced by TNF-alpha requires AKT/ GSK-3beta-mediated stabilization of snail in colorectal cancer. Plos One. 2013; 8:e56664.

38. Wu YM, Chen ZJ, Liu H, Wei WD, Lu LL, Yang XL, Liang WT, Liu T, Liu HL, Du J, Wang HS. Inhibition of ERRalpha suppresses epithelial mesenchymal transition of triple negative breast cancer cells by directly targeting fibronectin. Oncotarget. 2015. doi: 10.18632/oncotarget.4436.

39. Yao S, Zheng P, Wu H, Song LM, Ying XF, Xing C, Li Y, Xiao ZQ, Zhou XN, Shen T, Chen L, Liu YH, Lai MD, Mei L, Gao TM, Li JM. Erbin interacts with c-Cbl and promotes tumourigenesis and tumour growth in colorectal cancer by preventing c-Cbl-mediated ubiquitination and downregulation of EGFR. J Pathol. 2015; 236:65-77. 
40. Chen ZJ, Yang XL, Liu H, Wei W, Zhang KS, Huang HB, Giesy JP, Liu HL, Du J, Wang HS. Bisphenol A modulates colorectal cancer protein profile and promotes the metastasis via induction of epithelial to mesenchymal transitions. Arch Toxicol. 2015; 89:1371-1381

41. Jiang GM, Wang HS, Zhang F, Zhang KS, Liu ZC, Fang R, Wang H, Cai SH, Du J. Histone deacetylase inhibitor induction of epithelial-mesenchymal transitions via up-regulation of Snail facilitates cancer progression. BBAMol Cell Res. 2013; 1833:663-671.
42. Ge LC, Chen ZJ, Liu HY, Zhang KS, Liu H, Huang HB, Zhang G, Wong CK, Giesy JP, Du J, Wang HS. Involvement of activating ERK $1 / 2$ through $G$ protein coupled receptor 30 and estrogen receptor alpha/beta in low doses of bisphenol A promoting growth of Sertoli TM4 cells. Toxicol Lett. 2014; 226:81-89.

43. Wei W, Chen ZJ, Zhang KS, Yang XL, Wu YM, Chen XH, Huang HB, Liu HL, Cai SH, Du J, Wang HS. The activation of $\mathrm{G}$ protein-coupled receptor 30 (GPR30) inhibits proliferation of estrogen receptor-negative breast cancer cells in vitro and in vivo. Cell Death Dis. 2014; 5:e1428. 